\title{
Cerebral venous sinus thrombosis after vestibular schwannoma surgery: a call for evidence-based management guidelines
}

\author{
Hussam Abou-Al-Shaar, MD, ${ }^{1}$ Yair M. Gozal, MD, PhD, ${ }^{1}$ Gmaan Alzhrani, MD, ${ }^{1}$ \\ Michael Karsy, MD, PhD, MSc, ${ }^{1}$ Clough Shelton, MD, ${ }^{2}$ and William T. Couldwell, MD, PhD' \\ ${ }^{1}$ Department of Neurosurgery, Clinical Neurosciences Center; and 2Department of Surgery, Division of Otolaryngology, University \\ of Utah, Salt Lake City, Utah
}

OBJECTIVE Postoperative cerebral venous sinus thrombosis (CVST) is an uncommon complication of posterior fossa surgery. The true incidence of and optimal management strategy for this entity are largely unknown. Herein, the authors report their institutional incidence and management experience of postoperative CVST after vestibular schwannoma surgery.

METHODS The authors undertook a retrospective review of all vestibular schwannoma cases that had been treated with microsurgical resection at a single institution from December 2011 to September 2017. Patient and tumor characteristics, risk factors, length of stay, surgical approaches, sinus characteristics, CVST management, complications, and follow-up were analyzed.

RESULTS A total of 116 patients underwent resection of vestibular schwannoma. The incidence of postoperative CVST was $6.0 \%$ (7 patients). All 7 patients developed lateral CVST ipsilateral to the lesion. Four cases occurred after translabyrinthine approaches, 3 occurred after retrosigmoid approaches, and none occurred following middle cranial fossa approaches. Patients were managed with anticoagulation or antiplatelet therapy. Although patients were generally asymptomatic, one patient experienced intraparenchymal hemorrhage, epidural hemorrhage, and obstructive hydrocephalus, likely as a result of the anticoagulation therapy. However, all 7 patients had a modified Rankin scale score of 1 at the last follow-up.

CONCLUSIONS Postoperative CVST is an infrequent complication, with an incidence of $6.0 \%$ among 116 patients who had undergone vestibular schwannoma surgery at one institution. Moreover, the management of postoperative CVST with anticoagulation therapy poses a serious dilemma to neurosurgeons. Given the paucity of reports in the literature and the low incidence of CVST, additional studies are needed to better understand the cause of thrombus formation and help to establish evidence-based guidelines for CVST management and prevention.

https://thejns.org/doi/abs/10.3171/2018.4.FOCUS18112

KEYWORDS acoustic neuroma; vestibular schwannomas; retrosigmoid; translabyrinthine; middle cranial fossa; cerebral venous sinus thrombosis; evidence-based medicine; complication

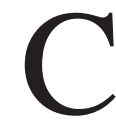

EREBRAL venous sinus thrombosis (CVST) primarily occurs in the setting of trauma, infection, and congenital or acquired hypercoagulable states, but nearly $20 \%-25 \%$ of CVST cases occur without an identifiable cause. ${ }^{16,19}$ It is estimated that $4.7 \%-11.6 \%$ of patients undergoing posterior fossa surgery for cerebellopontine angle tumors will develop postoperative lateral sinus thrombosis. ${ }^{1,8,11}$ With the relatively low incidence of this serious complication, there is a paucity of reports in the literature regarding the diagnosis, management, and sequelae associated with postoperative CVST.

To date, the management strategy applied in the treatment of postoperative thrombosis has been elucidated from the better-studied approaches used to treat spontaneously occurring CVST.,15 However, because the primary strategy for treating spontaneous CVST is aggressive an-

ABBREVIATIONS ASA = acetylsalicylic acid CTV = CT venography; $C V S T=$ cerebral venous sinus thrombosis; $E V D=$ external ventricular drain; LOS = length of stay; $\mathrm{mRS}=$ modified Rankin Scale; MRV = MR venography.

SUBMITTED February 28, 2018. ACCEPTED April 25, 2018.

INCLUDE WHEN CITING DOI: 10.3171/2018.4.FOCUS18112. 
ticoagulation, its application in the immediate postoperative period poses a serious dilemma for otologists and neurosurgeons. Specifically, surgeons must balance the risk of postoperative hemorrhage caused by venous hypertension associated with sinus thrombosis with the risk of hemorrhage due to perioperative anticoagulation. In the absence of evidence-based guidelines for the management of this iatrogenic entity, we report herein our experience with CVST after the resection of vestibular schwannomas.

\section{Methods}

We utilized the Enterprise Data Warehouse database at the University of Utah Health Sciences Center and a cross-referenced electronic medical record to identify patients who had undergone resection of a pathologically confirmed vestibular schwannoma during the period from September 2011 through September 2017. Patients treated with microsurgical resection of vestibular schwannomas were cross-referenced with an internal database maintained by the senior author (W.T.C.) from December 2007 to September 2017. Screening of these patients was accomplished by employing filters based on the ICD-10 code D33.3 (benign neoplasm of cranial nerves). Study exclusion criteria included an age $<18$ years or not having an elective procedure for the treatment of vestibular schwannoma. A manual chart review was performed to verify patients' inclusion and obtain clinical variables.

Microsurgical cases included those performed via retrosigmoid, translabyrinthine, or middle cranial fossa approaches. Selection of the applied surgical approach was dependent on specific patient and tumor characteristics, such as tumor size and location and preoperative hearing function, at the discretion of the senior authors (C.S. and W.T.C.).

The following variables were collected from the electronic medical records of the patients: age, sex, hypercoagulable risk factors (i.e., history of cancer, smoking, previous deep venous thrombosis, medications), tumor size, tumor site, Koos grade, ${ }^{9}$ surgical approach, length of stay (LOS), intraoperative direct cerebral venous sinus injury, development of postoperative CVST, transverse-sigmoid sinus dominance, CVST management, modified Rankin Scale (mRS) score ${ }^{2}$ at the last follow-up, and follow-up duration and status. The diagnosis of CVST was made on routine postoperative $\mathrm{CT}$ scan and confirmed with $\mathrm{CT}$ venography (CTV) or MR venography (MRV). Continuous variables are reported as the means \pm standard deviations, whereas percentages were used for categorical variables.

\section{Results}

\section{Patient and Surgical Characteristics}

During the study period, a total of 116 patients underwent resection of a pathologically confirmed vestibular schwannoma and were included in our analysis (Table 1). Among these patients were $60(51.7 \%)$ men and 56 (48.3\%) women; the mean age was $49.0 \pm 13.2$ years (range 18.075.4 years). The mean tumor size was $2.1 \pm 1.2 \mathrm{~cm}$ (range $0.4-4.4 \mathrm{~cm}$ ), and tumors occurred on the left side in 64 (55.2\%) patients and on the right side in 52 (44.8\%) pa-
TABLE 1. Summary of characteristics of 116 patients who underwent surgical removal of a vestibular schwannoma

\begin{tabular}{cc}
\hline \multicolumn{1}{c}{ Parameter } & Value \\
\hline Sex & $60(51.7 \%)$ \\
Male & $56(48.3 \%)$ \\
Female & $49.0 \pm 13.2$ \\
\hline Age in yrs & $2.1 \pm 1.2$ \\
\hline Tumor size in cm & \\
Tumor location & $52(44.8 \%)$ \\
Rt side & $64(55.2 \%)$ \\
Lt side & \\
Koos grade & $35(30.2 \%)$ \\
I & $33(28.4 \%)$ \\
II & $11(9.5 \%)$ \\
III & $37(31.9 \%)$ \\
IV & \\
\hline Surgical approach & $52(44.8 \%)$ \\
Translabyrinthine & $31(26.7 \%)$ \\
Retrosigmoid & $33(28.4 \%)$ \\
Middle cranial fossa & $5.0 \pm 3.5$ \\
\hline LOS in days & $16.6 \pm 24.3$ \\
\hline FU in mos &
\end{tabular}

$\mathrm{FU}=$ follow-up.

Values expressed as the mean \pm standard deviation or as number $(\%)$.

tients. Thirty-five (30.2\%) tumors were classified as Koos grade I, $33(28.4 \%)$ as Koos grade II, $11(9.5 \%)$ as Koos grade III, and 37 (31.9\%) as Koos grade IV. The translabyrinthine approach was employed in $52(44.8 \%)$ patients, the middle cranial fossa approach in 33 (28.4\%), and the retrosigmoid approach in $31(26.7 \%)$.

The mean LOS was $5.0 \pm 3.5$ days (range $2-25$ days). The mean follow-up was $16.6 \pm 24.3$ months (range $0-120.8$ months). At their last follow-up, $11.2 \%$ of patients had an $\mathrm{mRS}$ score $\geq 2$.

\section{Cerebral Venous Sinus Injury and Thrombosis}

Postoperative CVST occurred in 7 patients, resulting in an overall incidence of $6 \%$ in our study. Of these cases, the retrosigmoid approach was used in $3(10 \%)$ of 31 patients, whereas $4(8 \%)$ of 52 patients underwent a translabyrinthine approach. Two patients incurred an iatrogenic cerebral venous sinus injury that was repaired during surgery, although one of these patients also developed a postoperative CVST (Table 2). The other patient had a cerebral venous sinus injury but did not develop CVST, as outlined in Table 2 (case 8). Among the 7 patients with CVST, 5 were men and 2 were women. Their mean tumor size was $3.0 \pm$ $1.3 \mathrm{~cm}$ (range 1.2-5.0 $\mathrm{cm}$ ). The tumor was located on the left side in 4 patients and on the right side in 3 patients. Two patients had Koos grade II tumors, 2 had Koos grade III, and 3 had Koos grade IV. None of the patients developed postoperative CVST after a middle cranial fossa approach.

All 7 patients developed postoperative CVST ipsilateral to the lesion. Two patients had postoperative transverse and sigmoid sinus thrombosis, 1 had transverse-sigmoid junction thrombosis, 1 had sigmoid sinus thrombosis only, 
TABLE 2. Cerebral venous sinus injury and thrombosis patients

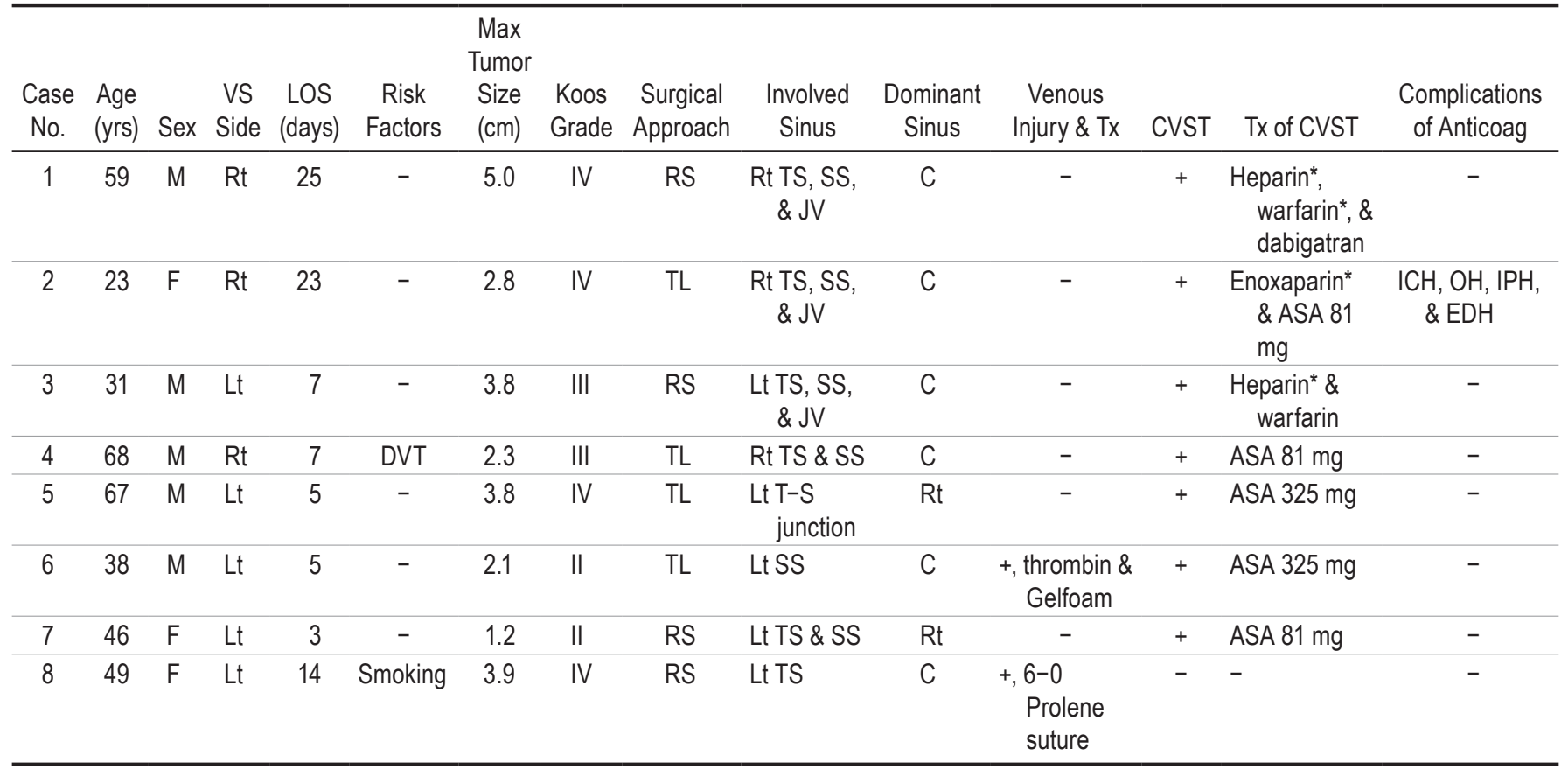

Anticoag = anticoagulation; $\mathrm{C}=$ co-dominant; $\mathrm{DVT}=$ deep venous thrombosis; $\mathrm{EDH}=$ epidural hematoma; $\mathrm{ICH}=$ intracranial hematoma; $\mathrm{IPH}=$ intraparenchymal hematoma; JV = jugular vein; $\mathrm{OH}$ = obstructive hydrocephalus; $\mathrm{RS}$ = retrosigmoid approach; $\mathrm{SS}=$ sigmoid sinus; $\mathrm{TL}$ = translabyrinthine approach; $\mathrm{T}-\mathrm{S}=$ transverse-sigmoid; $\mathrm{TS}=$ transverse sinus; $\mathrm{Tx}$ = treatment; $\mathrm{VS}$ = vestibular schwannoma; + = present; - = absent.

* Discontinued before discharge.

and 3 had thrombosis extending from the transverse sinus distally into the internal jugular vein. Sinus co-dominance was present in 5 patients, and in 2 patients the CVST involved the nondominant sinus. Only 1 patient had hypercoagulable risk factors (history of deep venous thrombosis), for which he completed a course of anticoagulation. None of the patients were specifically symptomatic from the CVST. In each case, CVST was detected on routine postoperative imaging and subsequently confirmed with CTV or MRV.

Management of the 7 patients experiencing CVST is outlined in Table 2. All patients received aggressive hydration to maintain an intravenous fluid rate of $40-50 \mathrm{ml} /$ $\mathrm{kg} /$ day. Four patients were started on antiplatelet therapy with aspirin (ASA), 1 was started on intravenous heparin with bridging to warfarin, 1 was started on therapeutic enoxaparin while an inpatient and ultimately discharged on ASA, and 1 underwent inpatient heparin bridging to warfarin but was discharged on dabigatran. Total duration of treatment ranged from 3 to 6 months. All patients were maintained on higher than maintenance rates of intravenous fluids until discharge. Notably, the patient initiated directly on enoxaparin therapy developed an extraaxial hemorrhage involving the cerebellopontine angle and cervicomedullary junction, an intraventricular hematoma, and concomitant obstructive hydrocephalus following the initiation of anticoagulation. As a result, therapy with enoxaparin was reversed with protamine sulfate (Fig. 1) and a right frontal external ventricular drain (EVD) was placed emergently for progressive ventriculomegaly. Drain placement was further complicated by epidural and intraparenchymal hematomas at the site of the procedure, requiring a right frontal craniotomy for evacuation of the extraaxial hemorrhage. Given the patient's complications in the setting of full-dose anticoagulation, she was ultimately maintained on antiplatelet therapy only. She was discharged to an inpatient rehabilitation unit but had only minimal gait instability and mild diplopia at the last follow-up. The remaining patients who underwent CVST treatment did not experience any complications.

The mean LOS among the 7 patients with CVST was $11.0 \pm 9.2$ days (range 3-25 days). The mean follow-up was $10.8 \pm 14.5$ months (range $0-120.8$ months). At their last follow-up, all 7 patients had an mRS score of 1 .

\section{Discussion}

Patients undergoing posterior fossa surgery for cerebellopontine angle tumors are at an increased risk for the development of CVST. ${ }^{1,11}$ In these cases, the treating physician is left to balance the risk of thrombotic complications with the hemorrhagic risks of anticoagulation in the immediate postoperative period. In the current study, we retrospectively reviewed our experience with CVST in patients undergoing microsurgical resection of vestibular schwannomas.

\section{Incidence of CVST After Posterior Fossa Surgery}

Previous studies have documented a rate of CVST of $4.7 \%-11.6 \%$ among patients undergoing posterior fossa surgery. ${ }^{1,8,11}$ In one of the largest series reported to date, Apra et al. ${ }^{1}$ analyzed the risk of postoperative lateral 

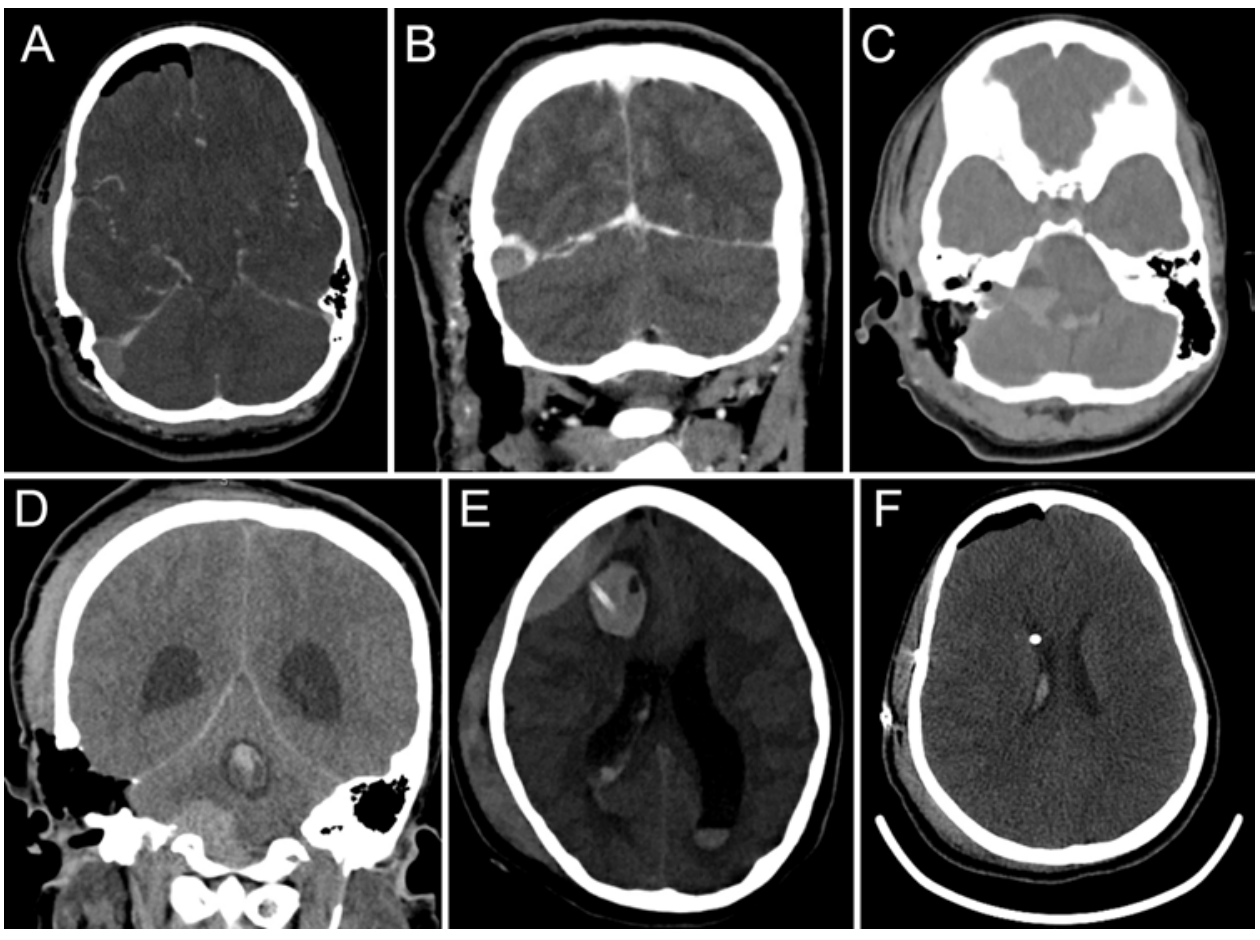

FIG. 1. Case 2. Axial (A) and coronal (B) CT venograms demonstrating right transverse sinus, sigmoid sinus, and internal jugular vein thrombosis after a translabyrinthine approach for vestibular schwannoma resection. Axial (C) and coronal (D) CT scans depicting cerebellopontine angle, cervicomedullary junction, and intraventricular hematoma leading to obstructive hydrocephalus. Axial CT scan (E) showing intraparenchymal and epidural hematomas following placement of an EVD, along with a midline shift. Axial CT scan (F) demonstrating successful hematoma evacuation and resolution of the ventriculomegaly and midline shift.

CVST among 180 patients who underwent suboccipital craniotomy for various cerebellopontine angle tumors, including meningiomas, gliomas, schwannomas, and metastatic lesions. The authors found that 12 patients $(6.7 \%$, 95\% CI 3.0-10.4) developed postoperative lateral CVST. In all patients, sinus thromboses occurred ipsilateral to the lesion, and none of the patients developed associated clinical symptomatology. Similarly, Keiper and colleagues ${ }^{8}$ reported postoperative lateral CVST in $4.7 \%$ of 107 patients, and Moore et al. ${ }^{11}$ reported a rate of $11.6 \%$ in 43 patients undergoing suboccipital or translabyrinthine approaches to the posterior fossa. In accordance with these reported incidences, our CVST rate was $6.0 \%$ in 116 patients with vestibular schwannomas treated via resection. As the above-mentioned studies included lesions of various histopathologies in their analyses, to the best of our knowledge, our study constitutes the first and largest study specifically examining postoperative CVST in patients with vestibular schwannomas.

\section{Symptoms Associated With CVST}

Patients with CVST may present with headaches, vision changes, seizures, altered mental status, intracranial hemorrhage, and other neurological sequelae., ${ }^{1,8,11,18}$ Whereas Keiper et al. ${ }^{8}$ reported the onset of headaches and visual changes in a delayed fashion in all 5 patients with CVST, the patients in our study remained entirely asymptomatic from their thrombotic complications. Our experience is supported by the studies by Apra et al. ${ }^{1}$ and Moore et al.;1 in the latter study, only one patient developed ataxia and cerebellar symptoms that resolved after CVST treatment. The clinical tolerance of postoperative CVST may be attributed to the presence of collateral venous channels or a dominant contralateral sinus. Moreover, it is possible that in a majority of patients, postoperative CVST may be an inherently less aggressive pathological process than spontaneous CVST, as the latter population is composed only of patients who have developed sufficiently severe symptoms to seek medical care. Nevertheless, surgeons should maintain a high index of suspicion after posterior fossa surgery to avoid missing postoperative CVST, particularly in asymptomatic patients. Routine postoperative CT screening is recommended.

\section{Risk of CVST With Surgery}

The transverse and sigmoid sinuses are the most commonly involved structures in CVST occurring after posterior fossa surgery. ${ }^{1,8,10,11}$ As in the current study, the majority of previously reported postoperative CVST cases occurred in these lateral cerebral venous sinuses ipsilateral to the lesion. ${ }^{1,10,11}$ Thrombotic complications involving the sinuses appear to correlate with the need for sinus exposure in these approaches. Accordingly, we found that patients who underwent retrosigmoid or translabyrinthine approaches in our study were most at risk for this complication, whereas those who were treated via the middle cranial fossa approach, in which the venous sinuses are not encountered (although the superior petrosal sinus is exposed in all cas- 
es), experienced no thrombotic complications. Although we found a slightly higher incidence of CVST after the retrosigmoid approach (10\%) compared with that after the translabyrinthine approach (8\%), it is unclear whether the degree of sinus exposure (partial exposure in retrosigmoid approach vs complete exposure in translabyrinthine approach) accounts for this variation in the incidence of postoperative CVST. Interestingly, all the cases of CVST in this series occurred in a co-dominant or nondominant sinus but not in a dominant sinus. This observation may be related to a higher blood flow in the dominant sinus in comparison with nondominant and superficial venous drainage patterns. Higher throughput of blood in the dominant side may decrease the risk for venous stasis, thrombus formation, and thrombus propagation postoperatively. In addition to mechanical sinus injury, numerous reported factors are thought to promote CVST formation. Among them are processes that contribute to dural desiccation of the sinus, including direct heat conduction during bony drilling and thermal radiation from the operative microscope lamp. ${ }^{1,8,11,13}$ To help mitigate these potential risk factors, efforts should be made to minimize sinus exposure and manipulation. Drilling around the sinus should be accomplished under constant irrigation, while exposed aspects of the sinus should be protected with moist neurosurgical patties. Retraction of the sinus should generally be avoided or frequently released to prevent intraluminal venous stasis. Finally, in the case of direct sinus injury, repair should be undertaken using nonabsorbable monofilament sutures (e.g., 6-0 Prolene suture).

\section{Management of CVST Associated With Surgery}

There remains significant heterogeneity among surgeons regarding the management of postoperative CVST primarily owing to the paucity of published reports and the relatively low incidence of this complication. Given the well-reported management of spontaneous CVST,,15 anticoagulation aimed at reducing the risk of sinus thrombosis propagation and the development of venous infarction is the mainstay of therapy for postsurgical cases of CVST. ${ }^{1,11}$ Apra et al. ${ }^{1}$ used therapeutic enoxaparin in a majority of patients, ultimately switching 1 patient to oral anticoagulation at discharge and electing to withhold anticoagulation from another with a small thrombus. Interestingly, these authors found a statistically significant elevated risk of surgical complications in anticoagulated patients with postoperative lateral CVST compared with that in patients without indication for anticoagulation $(\mathrm{p}=$ 0.020). However, none of these complications were related to post-anticoagulation intracranial hemorrhage. Moore et al. ${ }^{11}$ reported the successful management of postoperative CVST with the use of intravenous heparin transitioned to warfarin for 6 months in 5 patients. In their series, only 1 patient experienced mild vaginal bleeding as a complication of the anticoagulation therapy. Despite these reports, concerns for an increased risk of catastrophic hemorrhage in the acute postoperative period are rampant. ${ }^{13,14}$ In fact, in our own study, a CVST patient treated with enoxaparin experienced severe hemorrhagic complications attributable to therapeutic anticoagulation in the immediate postoperative period.
Given the limited data in the literature regarding the natural history of postoperative CVST, there exists significant variability in the management of this complication. In the absence of established guidelines, our management strategy for these patients is based on the experience of the senior author. As a matter of course, all patients undergoing presigmoid (translabyrinthine) or retrosigmoid approaches for resection of vestibular schwannomas should be aggressively hydrated in the perioperative period. In fact, many of our patients are now routinely maintained at an intravenous fluid rate of $40-50 \mathrm{ml} / \mathrm{kg} / \mathrm{day}$, rather than the standard maintenance rate of $25-30 \mathrm{ml} / \mathrm{kg} /$ day, for up to 3 days postoperatively. For patients with a direct intraoperative sinus injury requiring repair, we recommend the prophylactic initiation of antiplatelet therapy with ASA, usually started in the immediate postoperative period. The use of ASA in multiple patients was well tolerated in the current study, and it has been associated with limited risk when started in the immediate perioperative period in neurosurgical patients. ${ }^{7}$ After injury, the goal in using ASA is to prevent intraluminal thrombus or propagation of the clot if intraluminal thrombus occurs. In patients with CTV- or MRV-confirmed CVST after its identification on routine postoperative $\mathrm{CT}$, if the sinus is dominant or co-dominant, we have utilized therapeutic anticoagulation with intravenous heparin but without initial bolus dosing. Although many patients with postoperative CVST may remain asymptomatic without treatment, we have experienced complications with untreated CVST. The sequelae of postoperative CVST can be devastating, including a venous infarct, seizures, hydrocephalus, or even death. ${ }^{1,8}$ Thus, we use anticoagulation with heparin in all patients with radiographically documented sinus thrombosis, unless it is demonstrated that the involved sinus is diminutive in comparison to the contralateral patent sinus and the sinuses communicate at the torcula. If a postoperative CVST is symptomatic, it is treated with anticoagulation in this manner regardless of dominance. Therapeutic low-molecular-weight heparin (LMWH) is avoided in the early postoperative period. Patients unresponsive to anticoagulation therapy, as demonstrated by progression on repeat CTV or MRV 3-7 days after the initiation of treatment, or those who demonstrate acute neurological deterioration are candidates for more aggressive measures. Notably, reports of successful endovascular treatment of CVST with thrombolytics and mechanical thrombolysis are emerging. ${ }^{4-6,10,12,17}$ In the absence of progression on repeat venous imaging, the patient may be transitioned to either oral anticoagulation or therapeutic enoxaparin for a 3- to 6-month period.

\section{Limitations and Future Directions}

The retrospective single-site study design and the low incidence of postoperative CVST represent our primary study limitations. Although our institution is a high-volume center for vestibular schwannomas, our reliance on noncontrasted CT scans as the first-line screening for CVST likely further limited the study sample size. Ultimately, multicenter prospective studies employing a single management strategy may be required to establish appropriate treatment guidelines for postoperative CVST. 


\section{Conclusions}

Postoperative CVST is an uncommon complication following vestibular schwannoma surgery. In our series of 116 patients treated with microsurgical resection, postoperative CVST occurred in $6.0 \%$ of patients and was managed with either antiplatelet or anticoagulation therapy. Because of the lack of evidence-based guidelines, the management of postoperative CVST is challenging. Although we present our management strategy, future prospective studies are needed to better understand CVST, its optimal management, and its long-term sequelae. Although anticoagulation of CVST has inherent risks, as demonstrated by the clinical course of the patient whose imaging studies are shown in Fig. 1, untreated CVST of a dominant or codominant sinus is also associated with the risk of neurological deterioration. We plan to address this issue with a prospective study.

\section{Acknowledgments}

We thank Kristin Kraus, MSc, for her editorial assistance.

\section{References}

1. Apra C, Kotbi O, Turc G, Corns R, Pagès M, Souillard-Scémama R, et al: Presentation and management of lateral sinus thrombosis following posterior fossa surgery. $\mathbf{J}$ Neurosurg 126:8-16, 2017

2. Bonita R, Beaglehole R: Modification of Rankin scale: recovery of motor function after stroke. Stroke 18:1497-1500, 1988

3. Ferro JM, Canhão P, Stam J, Bousser MG, Barinagarrementeria F: Prognosis of cerebral vein and dural sinus thrombosis: results of the International Study on Cerebral Vein and Dural Sinus Thrombosis (ISCVT). Stroke 35:664-670, 2004

4. Froehler MT: Successful treatment of cerebral venous sinus thrombosis with the Solitaire FR thrombectomy device. J Neurointerv Surg 5:e45, 2013

5. Gala NB, Agarwal N, Barrese J, Gandhi CD, Prestigiacomo CJ: Current endovascular treatment options of dural venous sinus thrombosis: a review of the literature. J Neurointerv Surg 5:28-34, 2013

6. Guo XB, Guan S, Fan Y, Song LJ: Local thrombolysis for severe cerebral venous sinus thrombosis. AJNR Am J Neuroradiol 33:1187-1190, 2012

7. Kamenova M, Lutz K, Schaedelin S, Fandino J, Mariani L, Soleman J: Does early resumption of low-dose aspirin after evacuation of chronic subdural hematoma with burr-hole drainage lead to higher recurrence rates? Neurosurgery 79:715-721, 2016

8. Keiper GL Jr, Sherman JD, Tomsick TA, Tew JM Jr: Dural sinus thrombosis and pseudotumor cerebri: unexpected complications of suboccipital craniotomy and translabyrinthine craniectomy. J Neurosurg 91:192-197, 1999

9. Koos WT, Day JD, Matula C, Levy DI: Neurotopographic considerations in the microsurgical treatment of small acoustic neurinomas. J Neurosurg 88:506-512, 1998

10. Manzoor NF, Ray A, Singer J, Nord R, Sunshine J, Megerian CA, et al: Successful endovascular management of venous sinus thrombosis complicating trans-labyrinthine removal of vestibular schwanomma. Am J Otolaryngol 37:379-382, 2016
11. Moore J, Thomas P, Cousins V, Rosenfeld JV: Diagnosis and management of dural sinus thrombosis following resection of cerebellopontine angle tumors. J Neurol Surg B Skull Base 75:402-408, 2014

12. Nimjee SM, Powers CJ, Kolls BJ, Smith T, Britz GW, Zomorodi AR: Endovascular treatment of venous sinus thrombosis: a case report and review of the literature. J Neurointerv Surg 3:30-33, 2011

13. Ohata K, Haque M, Morino M, Nagai K, Nishio A, Nishijima $\mathrm{Y}$, et al: Occlusion of the sigmoid sinus after surgery via the presigmoidal-transpetrosal approach. J Neurosurg 89:575584, 1998

14. Sawarkar DP, Verma SK, Singh PK, Doddamani R, Kumar A, Sharma BS: Fatal superior sagittal sinus and torcular thrombosis after vestibular schwannoma surgery: report of a rare complication and review of the literature. World Neurosurg 96:607.e19-607.e24, 2016

15. Stam J, de Bruijn S, deVeber G: Anticoagulation for cerebral sinus thrombosis. Stroke 34:1054-1055, 2003

16. Steven A, Raghavan P, Altmeyer W, Gandhi D: Venous thrombosis: causes and imaging appearance. Hematol Oncol Clin North Am 30:867-885, 2016

17. Velat GJ, Skowlund CJ, Waters MF, Mocco J, Hoh BL: Direct thrombectomy using the Penumbra thromboaspiration catheter for the treatment of cerebral venous sinus thrombosis. World Neurosurg 77:591.e15-591.e18, 2012

18. Wasay M, Kojan S, Dai AI, Bobustuc G, Sheikh Z: Headache in cerebral venous thrombosis: incidence, pattern and location in 200 consecutive patients. J Headache Pain 11:137139,2010

19. Zuurbier SM, Coutinho JM: Cerebral venous thrombosis. Adv Exp Med Biol 906:183-193, 2017

\section{Disclosures}

The authors report no conflict of interest concerning the materials or methods used in this study or the findings specified in this paper.

\section{Author Contributions}

Conception and design: Couldwell, Abou-Al-Shaar, Gozal, Alzhrani, Shelton. Acquisition of data: Abou-Al-Shaar, Alzhrani, Karsy. Analysis and interpretation of data: Abou-Al-Shaar, Gozal, Karsy. Drafting the article: Abou-Al-Shaar, Gozal. Critically revising the article: all authors. Reviewed submitted version of manuscript: all authors. Approved the final version of the manuscript on behalf of all authors: Couldwell. Study supervision: Couldwell.

\section{Supplemental Information Current Affiliations}

Dr. Abou-Al-Shaar: Department of Neurosurgery, Hofstra Northwell School of Medicine, Manhasset, NY.

Dr. Alzhrani: Department of Neurosurgery, National Neuroscience Institute, King Fahad Medical City, Riyadh, Kingdom of Saudi Arabia.

\section{Correspondence}

William T. Couldwell: University of Utah, Salt Lake City, UT. neuropub@hsc.utah.edu. 